\title{
ZnS: Mn-SPP semiconductor nanocrystals: Synthesis and optical and structural characterization
}

\author{
A. Rahdar \\ ${ }^{1}$ Department of Physics, Faculty of Science, University of Zabol, Zabol, Iran. \\ a.rahdar@uoz.ac.ir
}

\begin{abstract}
The present paper reports the synthesis and characterization of Nano crystals of manganese doped zinc sulphide, which were prepared by co-precipitation method using the solution of zinc chloride, sodium sulphide and manganese chloride, whereas sodiumpolyphosphate(SPP) was used as an capping agent. The particle size of such nanocrystals was measured using XRD pattern and it is found to be in between 2.49-1.82. The optical absorption spectra of the samples obtained using UV-Vis spectrophotometer shows the blue-shift with decreasing particle size. We also found that optical band gap $(\mathrm{Eg})$ increases with the increase in molar concentration of doping agent. This behavior is related to size quantization effect due to the small size of the particles. PI spectrum of SPP-capped ZnS:Mn nanocrystals obtained by excitation at $285 \mathrm{~nm}$ shows a peak at $572 \mathrm{~nm}$. The particle sizes obtained from TEM images
\end{abstract}

Keywords: ZnS: Mn, Co-precipitation, Sodiumpolyphosphate, Capping agent, Optical band gap, pl spectrum.

\section{Introduction}

Semiconductor nanocrystals, so called quantum dots (QDs), are a newly emerging nanomaterial, which have attracted many interests. Having excitons confined in all three spatial dimensions and the increase of surface area to volume ratio, quantum dots have properties that are between those of bulk semiconductors and those of discrete molecules. When excited by photons or electric field with energy higher than the band gap, quantum dots can emit photons releasing the absorbed energy. Moreover, the emission energy is adjustable by controlling the composition and the particle size of quantum dots due to the quantum confinement effect. Due to the distinguished photoluminescence and electroluminescence properties, quantum dots have been explored extensively for various applications, including imaging, detection, therapy, display and energy harvesting, etc (Rossetti et al., 1984).

In this between, ZnS semiconductor nanocrystals has been used widely as a promising candidate for photoluminescence, electroluminescence and cathodoluminescence devices due to its better chemical stability compared to other chalcogenides such as $\mathrm{ZnSe}$. In optoelectronics, it is used as light emitting diode, reflector, dielectric filter and window material (Zhai et al., 2002). Research on nanocrystals of $\mathrm{ZnS}$ containing Mn, $\mathrm{Fe}, \mathrm{Ni}$ and $\mathrm{Cu}$ has been in full swing as the solubility limit for these transition metals in II-VI host lattice is high (Ping Yang et al., 2003; Warad et al., 2005; Sambasivam et al., 2008). Nevertheless the theoretical and experimental researches on $\mathrm{Mn}$ doped $\mathrm{ZnS}$ are still limited. There are different methods for synthesis and characterization of nanometer- sized semiconductors. The functionalization of surfaces is another important aspect of nanoparticle fabrication. In particular, it is important to make nanoparticles water-dispersible for many biological applications. Surfactants play important roles in the control of dispersed particle sizes, optical properties, and solubility by passivating nanocrystal surfaces (Dios et al., 2005). Here, we report on the synthesis and structural and optical properties of water-dispersible $\mathrm{Mn}$-doped $\mathrm{ZnS}$ nanoparticles stabilized by sodiumpolyphosphate (ZnS:Mn-SPP) as a capping agent, which modifies surface of nanoparticles and prevents the growth of the particles to larger size.

Materials and methods

Zinc chloride $(\mathrm{ZnCl} 2)$, sodium sulfide ( $\mathrm{Na} 2 \mathrm{~S})$ and $(\mathrm{MnCl} 2)$ as starting materials, sodiumpolyphosphate as a capping agent for control particles size and doubledistilled water as dispersing solvent were used to prepare ZnS:Mn nanoparticles (Rahdar et al., 2012).

The ZnS:Mn-SPP nanoparticles were prepared by the chemical co-precipitation method as follows. First, $\mathrm{ZnCl} 2$ $(50 \mathrm{ml})$ was dissolved in double-distilled water with 0.1 molar concentrations and then obtained molar so-lution was stirred for $20 \mathrm{~min}$ at room temperature to achieve complete dissolution. $\mathrm{Na} 2 \mathrm{~S}$ was also dissolved in doubledistilled water separately as per molar concentra-tion and volume. Afterwards, first $\mathrm{Na} 2 \mathrm{~S}$ solution was added drop by drop to the $\mathrm{ZnCl} 2$ solution. Next the $\mathrm{MnCl} 2(50 \mathrm{ml}$ ) solution with certain molar concentration was added to this solution. Then, an appropriate amount $(50 \mathrm{ml})$ of sodiumpolyphosphate $(0.1 \mathrm{M})$ was added to the reaction medium to control the particle size of $\mathrm{ZnS}: \mathrm{Mn}$. The resulting solution was stirred continuously for 6 hours. In the final step, the white obtained precipitate was filtered and dried at room temperature to remove both water and organic capping and other byproducts formed during the reaction process. After sufficient drying, the precipitate was crushed to fine powder with the help of mortar and pestle.

It is necessary to mention that different sample of nanoparticles has been obtained by changing the molar concentration of doping agent.
Research article

CCIndian Society for Education and Environment (iSee)
ZnS:MnSPP semiconductor nanocrystals http://www.indjst.org
A.Rahdar

Indian J.Sci.Technol. 


\section{Results and discussion}

The XRD patterns for of ZnS:Mn-SPP nanoparticles were recorded by Bruker system using $\mathrm{Cu} \mathrm{Ka}$ radiation $(\lambda=0.154056 \mathrm{~nm})$ with $2 \theta$ ranging $20-70^{\circ}$ And with cer-tain molar concentration of doping agent are shown in Fig. 1. The lower panel shows the XRD spectrum of bulk ZnS in a zinc blende for Three different peaks are obtained at $2 \theta$ values of $29.190,48.410$ and 57.580 for $\mathrm{ZnS}: \mathrm{Mn}-\mathrm{SSP}$ nanoparticles. This shows that the samples have zinc blende structure and the peaks correspond to diffraction at (111), (220) and (311) planes, respectively. The lattice parameter has been computed as $6.29 \AA$, which is close to the standard value $(5.42 \AA)$ ). It is also seen from the Fig. 1 that peaks are broadened. A careful observation of the figure reveals that the broadening occurs by increasing Molar concentration of doping agent.

Fig. 1. XRD spectrum of SPP-capped ZnS:Mn nanocrystals (Mn 3\%)

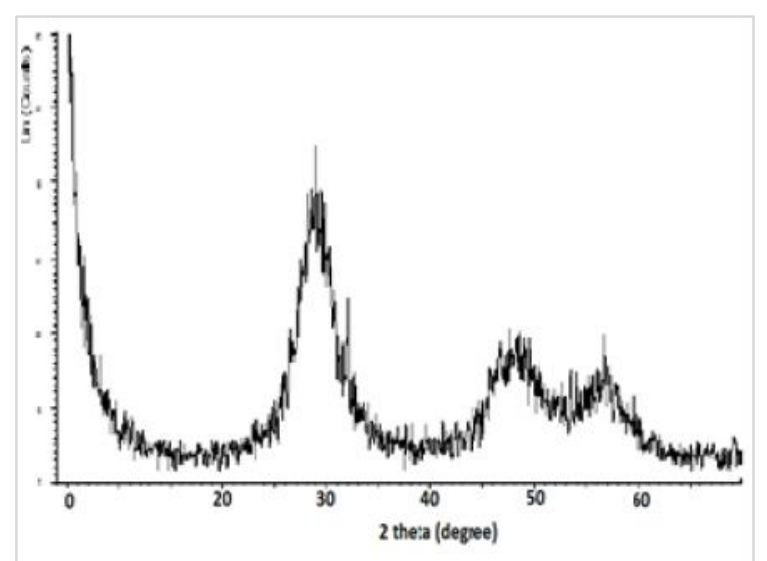

The peak broadening at lower angle is more meaningful for the calculation of particle size. The mean crystallite size (D) of nanoparticles was also estimated using the Scherrer formula using (111) reflection from the XRD pattern as follows:

$\mathrm{D}=\frac{0.9 \lambda}{\mathrm{B} \cos \theta}$,

Where $\lambda, B$, and $\theta$ are the $X$-ray wavelength of the radiation used $(\mathrm{Ka}(\mathrm{Cu})=0.154056 \mathrm{~nm})$, the full width at half maximum (FWHM) of the diffraction peak and the Bragg diffraction angle, respectively.

The optical absorption spectra of nanoparticles were measured using a USB-2000 UV-Vis spectrophotometer.The absorption spectra of the different samples are shown in Fig. 2. Fig. 2 shows the optical absorption spectra of ZnS nanoparticles with different concentration of $\mathrm{Mn}$ where as the concentration of capping agent remains same.The absorption edge is observed in the range of $320-280 \mathrm{~nm}$.

As the $\mathrm{Mn}$ concentration increases, the absorption edge shifts to lower wavelength side and intensity also increases with increasing $\mathrm{Mn}$ concentration compared to undoped ZnS. This blue shift of the absorption edges for different sized nanocrystals is related to the size decrease of particles and is attributed to the quantum

Fig. 2. Absorption spectra of ZnS:Mn-SPP nanoparticles with different molar concentration of doping agent (a: Mn 1\%) and b: (Mn 3\%)

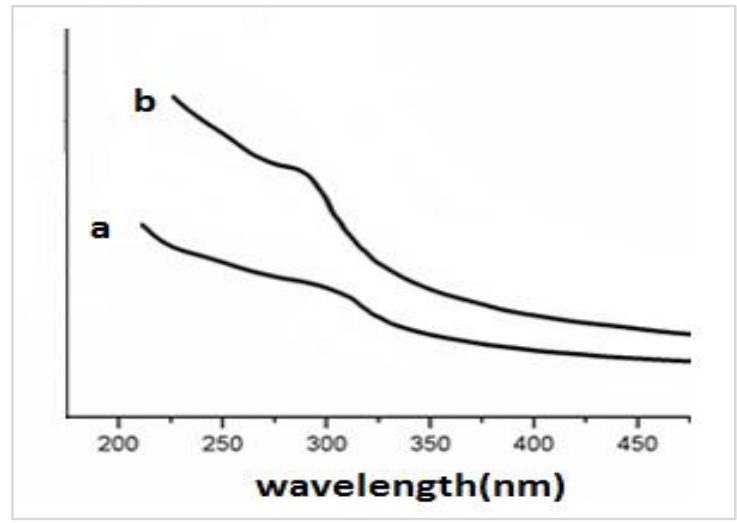

confinement effect of nanoparticles.The variation of optical band gap with particle size is shown in Fig. 3. The optical band gap values of nanoparticles have changed from 3.82 to $4.42 \mathrm{eV}$ by decreasing the particle sizes. The increase in band gap with increase in Mn concentration is attributed to size quantization effect due to the small size of the particles (Amaranatha et al., 2011)

The ZnS:Mn-SSP powder was highly soluble in water and was subjected to PI (Perkin-Elmer) spectroscopy in aqueous solution. Figure 4 shows the $\mathrm{PI}$ spectrum obtained by exciting at $285 \mathrm{~nm}$ (Fig. 4)

Despite the interference of light used for excitation, a $\mathrm{PI}$ band is clearly visible with a peak at $572 \mathrm{~nm}$. The large Stokes shift is attributed to the $4 \mathrm{~T} 1 \rightarrow 6 \mathrm{~A} 1$ transition of

Fig. 3. The variation of optical band gap

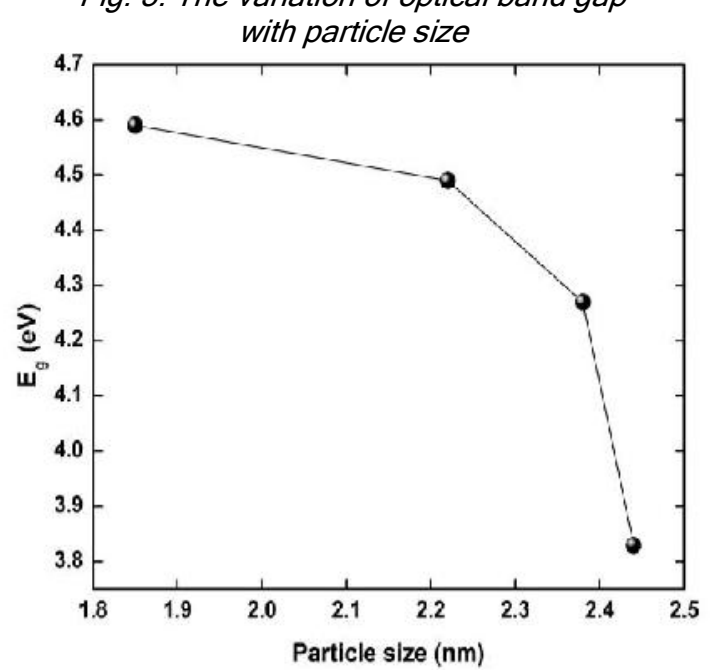

the Mn doping agent. In the other word,The peak at 572 $\mathrm{nm}$ arises due to the excitation and subsequent deexcitation of $\mathrm{Mn} 2+$ ions, Similar results are shown by Kim et al. (2008). 
These semiconductor nanocrystals were then subjected to transmission electron microscopy (TEM, JEOL JEM3010) for characterization. Fig. 5 shows TEM image of SPP-capped ZnS:Mn Semiconductor nanocrystals.

Fig. 4. PI spectrum of SPP-capped ZnS:Mn nano-crystals (Mn 3\%)

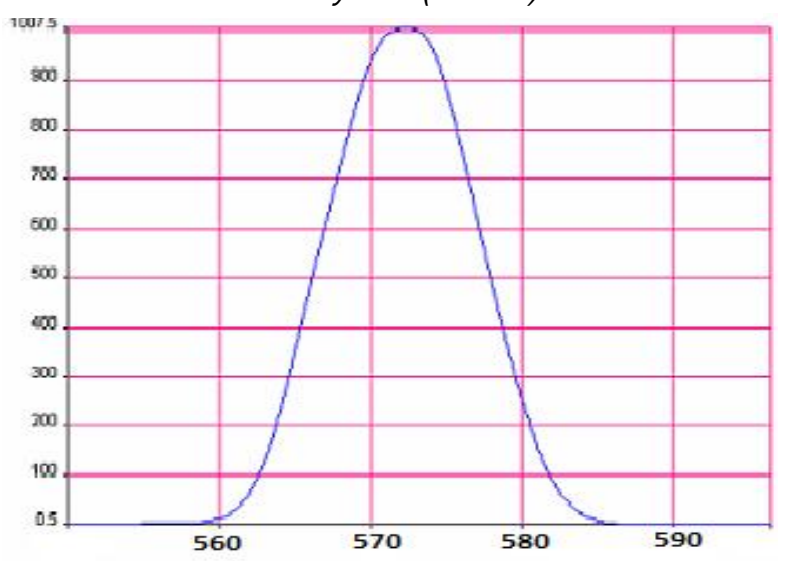

Fig. 5. TEM image of SPP-capped ZnS:Mn Semi-conductor nanocrvstals (Mn 3\%)

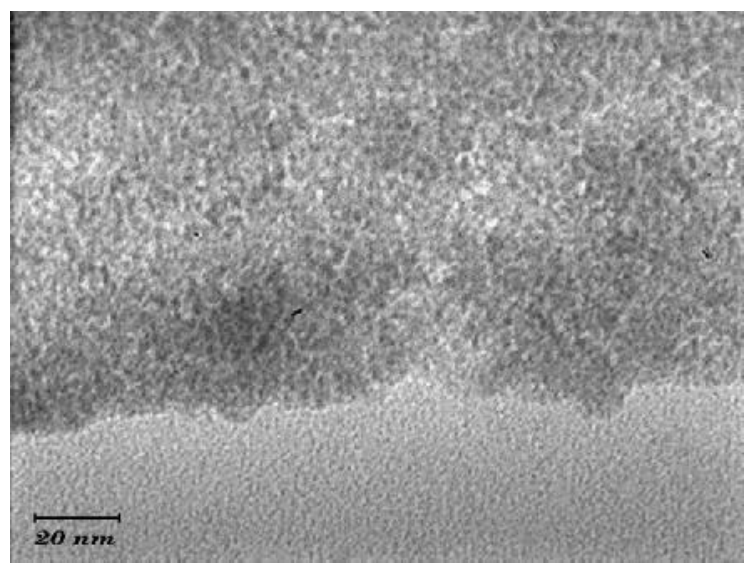

\section{Conclusions}

The nanoparticles of manganese doped zinc sulfide have been successfully synthesized by a simple precipitation reaction using different molar concentra-tion of doping agent in which sodiumpolyphosphate was used as a capping agent. It is also observed that the decrease in the size of particle occur with the increase of molar concentration of doping agent. XRD and Optical band gap data have been obtained to confirm nano-size of these materials. XRD analysis shows the sample prepared is in a cubical phase. The broad peak of XRD pattern indicates nanocrystalline behavior of the particles. The optical band gap values of $\mathrm{ZnS}: \mathrm{Mn}$ nanoparticles have changed from 3.81 to $4.24 \mathrm{eV}$ by increasing the molar concentration of doping agent. The absorption peak appears at around $280-320 \mathrm{~nm}$, which is fairly blueshifted from the absorption edge of the bulk (345 nm). The solid-state theory based on the delocalized electron and hole within the confined volume can explain the blueshifted optical absorption spectra. The PL peak $572 \mathrm{~nm}$ is indicating the $\mathrm{Mn} 2+$ ion in the orange-red region of the spectrum.TEM image shows clearly that the particles are not spherical.

\section{Acknowledgement}

The authors would like to thank Dr. M. Ali-Ahmad, Mrs.M.Asudeh,Mr.H.Rahdar and Mrs. Heidari Mokarrar for their support and assistance with this project.

\section{References}

1. Amaranatha Reddy D, Divya A, Murali G, Vijayalakshmi RP and Reddy B K (2011) Synthesis and optical properties of $\mathrm{Mn}$ doped $\mathrm{ZnS}$ nanoparticles capped by 2Mercaptoethanol. Physica. B406, 1944-1949.

2. Dios M de, Barroso F, Tojo C, Blanco M C and LopezQuintela M A (2005) Effects of the reaction rate on the size control of nanoparticles synthesized in microemulsions. Colloids \& Surfaces A: Physicochem. Engg. Aspects. 83, 270-271.

3. Ping Yang, Mengkai Lu, Dong $\mathrm{Xu}$, Duorong Yuan, Chunfeng Song, Suwen Liu and Xiufeng Cheng (2003) Luminescence characteristics of ZnS nanoparticles codoped with $\mathrm{Ni}^{2+}$ and $\mathrm{Mn}^{2+}$. Optical Materials. 24,497-502.

4. Rahdar A, Asnaasahri Eivari H and Sarhaddi R (2012) Study of structural and optical properties of $\mathrm{ZnS}: \mathrm{Cr}$ nanoparticles synthesized by co-precipitation method. Indian J. Sci. Technol. 5, 1855-1858.

5. Rossetti R, Ellison JL, Gibson JM and Brus LE (1984) Size effects in the excited electronic states of small colloidal CdS crystallites J. Chem Phys. 80, pp: 4464.

6. Sambasivam $S$, Paul Joseph $D$, Raja Reddy $D$, Reddy BK and Jayasankar C K (2008) Synthesis and characterization of thiophenol passivated Fe-doped ZnS nanoparticles. Mater. Sci. Engg. B150, 125-129.

7. Warad HC, Ghosh SC, Hemtanon B, Thanachayanont C and Dutt J (2005) Luminescent nanoparticles of $\mathrm{Mn}$ doped ZnS passivated with sodium hexametaphosphate. Sci. Technol. Adv. Mater. 6, pp: 296.

8. Zhai Q, Li J, Lewis JS, Waldrip KA, Jones K, Holloway $\mathrm{PH}$, Davidson Ma and Evans N (2002) Microstructure and electroluminescence of $\mathrm{ZnS}: \mathrm{Mn}$ doped with $\mathrm{KCl}$. Thin Solid Films. 414, 105-112.
A.Rahdar Indian J.Sci.Technol. 